\title{
Relativistic descriptions of quasielastic charged-current neutrino-nucleus scattering: Application to scaling and superscaling ideas
}

\author{
Andrea Meucci, ${ }^{1}$ J. A. Caballero, ${ }^{2}$ C. Giusti, ${ }^{1}$ and J. M. Udías ${ }^{3}$ \\ ${ }^{1}$ Dipartimento di Fisica Nucleare e Teorica, Università degli Studi di Pavia and Istituto Nazionale di Fisica Nucleare, \\ Sezione di Pavia, I-27100 Pavia, Italy \\ ${ }^{2}$ Departamento de Física Atómica, Molecular y Nuclear, Universidad de Sevilla, E-41080 Sevilla, Spain \\ ${ }^{3}$ Grupo de Física Nuclear, Departamento de Física Atómica, Molecular y Nuclear, Universidad Complutense de Madrid, \\ E-28040 Madrid, Spain
}

(Received 2 March 2011; revised manuscript received 4 May 2011; published 24 June 2011)

\begin{abstract}
The analysis of the recent experimental data on charged-current neutrino-nucleus scattering cross sections measured at MiniBooNE requires relativistic theoretical descriptions also accounting for the role of finalstate interactions. In this work we evaluate inclusive quasielastic differential neutrino cross sections within the framework of the relativistic impulse approximation. Results based on the relativistic mean-field potential are compared with the ones corresponding to the relativistic Green's-function approach. An analysis of scaling and superscaling properties provided by both models is also presented.
\end{abstract}

DOI: 10.1103/PhysRevC.83.064614

PACS number(s): 25.30.Pt, 25.30.Fj, 13.15.+g, 24.10.Jv

\section{INTRODUCTION}

From the analysis of the world $\left(e, e^{\prime}\right)$ data at quasielastic (QE) kinematics, i.e., with non-nucleonic degrees of freedom playing a minor role, has clearly emerged the validity of scaling arguments for values of the transferred momentum $q$ high enough [1-4]. Furthermore, the study of the separated longitudinal/transverse $(L / T)$ data has shown that whereas the $L$ response superscales, scaling violations are visible in the $T$ channel due to effects beyond the impulse approximation (IA): correlations, meson exchange currents, etc. These results constitute strong constraints for any theoretical model applied to electron scattering reactions; not only scaling and superscaling behavior should be fulfilled, i.e., independence on $q$ and on the nuclear target, but also the model should be capable to reproduce the specific shape of the experimental superscaling longitudinal function that presents a significant tail extended to large values of the transferred energy, that is, positive values of the superscaling variable $\psi$ [4].

A large variety of theoretical models used in the literature satisfy scaling arguments. This is the case of the relativistic Fermi gas (RFG) model that superscales by construction [2-4]. Other approaches based on nonrelativistic reductions also fulfill scaling behavior even in the presence of final-state interactions (FSI) and nucleon correlations (see Refs. [5-9]). However, most of them lead to scaling functions that do not get asymmetry, being in clear disagreement with data. Our past studies have shown that there are two basic aspects in the description of electron scattering that are necessary to compare superscaling properties of different models with experimental data: relativity and FSI. On the one hand, scaling comes out only when the momentum transfer is high enough. This means that not only relativistic kinematics should be considered, but also the nuclear dynamics and the description of the operators should involve relativity. On the other hand, the importance of FSI has also been clearly stated in the case of exclusive $\left(e, e^{\prime} N\right)$ processes where the use of complex optical potentials is required [10-18]. However, even being restricted to the
QE kinematical domain, the analysis of inclusive reactions, contrary to exclusive ones, needs all inelastic channels in the final state to be retained, i.e., the flux should be conserved. Therefore the distorted-wave impulse approximation based on the use of a complex potential should be dismissed for $\left(e, e^{\prime}\right)$ processes.

Previous studies [19-22] have clearly illustrated the key role played by FSI to reproduce the specific asymmetric shape shown by the experimental scaling function. Specifically, description of the final nucleon state using the same relativistic mean-field potential considered in describing the initial state, denoted simply as RMF, leads to a superscaling function in accordance with data. Recently [22], we have extended this analysis by introducing the relativistic Green's-function (RGF) technique developed by the Pavia group [23-26]. This is an alternative, relativistic approach to FSI in $\left(e, e^{\prime}\right)$ reactions. Both descriptions, RMF and RGF, contrary to previous models based on nonrelativistic or semirelativistic reductions [5], provide a significant asymmetry in the scaling function following the general behavior of data. It is also important to point out that asymmetry in the scaling function can be also obtained within the framework of a semirelativistic (SR) model, provided that FSI are described by the Dirac equation-based (DEB) potential derived from the RMF [9].

High-quality predictions for neutrino-nucleus cross sections are needed for use in ongoing experimental studies of neutrino oscillations at $\mathrm{GeV}$ energies. Recently, muon charged-current neutrino-nucleus double differential cross sections have been measured by the MiniBooNE collaboration [27]. The experimental conditions have made it possible to disentangle data for which no pions in the final state are detected. This corresponds to the $\mathrm{QE}$ regime where only nucleons are the relevant degrees of freedom, and is simply denoted as charged-current quasielastic (CCQE) neutrino-nucleus scattering. The analysis of CCQE data shows that the RFG model understimates the total cross section, unless the axial mass $M_{A}$ is significantly enlarged with 
respect to the accepted world average value. The kinematics involved in the MiniBooNE experiment, neutrino energy flux ranging from $375 \mathrm{MeV}$ up to $\sim 3 \mathrm{GeV}$, requires the use of a relativistic description of the process. Moreover, a proper analysis of data requires also good control on nuclear effects $[5,19,20,24,28-43]$. Notice that any reliable calculation for neutrino scattering should first be tested against electron scattering in the same kinematical conditions. In this sense, while the RFG is capable of getting the basic size and shape of the inclusive cross section, it can hardly account for important details of the response. Thus the need for a modification of the axial mass within the RFG could be a way to effectively incorporate nuclear effects into the calculation. As mentioned, relativity is expected to play an important role at MiniBooNE conditions. Not only relativistic kinematics, already included in the RFG model, but also dynamical relativistic effects, not present in RFG, can be important. In this work, the two QE-based approaches selected, RMF and RGF, incorporate relativistic dynamics by means of strong scalar and vector potentials in the Dirac equation employed to describe nucleons in nuclei.

As shown in Refs. [19-22], the RMF and RGF approaches describe successfully the behavior of electron-scattering data and their related scaling and superscaling functions. Therefore in this work we extend our previous analysis for $\left(e, e^{\prime}\right)$ reactions to charged-current $(\mathrm{CC})$ neutrino-nucleus scattering. The differences observed between the predictions of the two approaches can be helpful for understanding nuclear effects, particularly FSI, which may play a crucial role in the analysis of neutrino-nucleus scattering data and its influence in studies of neutrino oscillations at intermediate to high energies.

The paper is organized as follows. In Sec. II we summarize the basic formalism involved in the description of CC neutrinonucleus scattering. We introduce the main expressions and present a brief discussion on the two approaches considered in the description of final-state interactions, namely, the relativistic mean-field and the relativistic Green's-function technique. Scaling and superscaling arguments for neutrinonucleus scattering processes are also briefly mentioned. In Sec. III we show and discuss the results obtained with the two models considered. After checking the reliability of both calculations in the relativistic plane-wave approach and also making use of the real part of the relativistic optical potential, we describe FSI within the RMF and RGF models, and present results for the differential cross sections and scaling functions. Finally, in Sec. IV we summarize our conclusions.

\section{NEUTRINO-NUCLEUS SCATTERING}

The single-nucleon cross section for an inclusive reaction where an incident neutrino (antineutrino), with fourmomentum $k^{\mu}=\left(E_{v}, \boldsymbol{k}\right)$, is absorbed by a nucleus and only the outgoing muon, with four-momentum $k^{\prime \mu}=\left(\varepsilon^{\prime}, \boldsymbol{k}^{\prime}\right)$ and mass $m \simeq 105.7 \mathrm{MeV}$ is detected, is given by $[5,6,44,45]$

$$
\frac{d \sigma}{d \Omega^{\prime} d \varepsilon^{\prime}}=\sigma_{0} \mathcal{F}_{+(-)}^{2}
$$

where

$$
\sigma_{0}=\frac{\left(G \cos \vartheta_{C}\right)^{2}}{2 \pi^{2}} k^{\prime} \varepsilon^{\prime} \cos ^{2}(\bar{\vartheta} / 2)
$$

$G \simeq 1.166 \times 10^{-5} \mathrm{GeV}^{-2}$ is the Fermi constant, $\vartheta_{C}$ is the Cabibbo angle with $\cos ^{2}\left(\vartheta_{C}\right) \simeq 0.975$, and $\bar{\vartheta}$ is the generalized scattering angle, defined by

$$
\tan ^{2}(\bar{\vartheta} / 2)=\frac{\left|Q^{2}\right|}{\left(E_{v}+\varepsilon^{\prime}\right)^{2}-q^{2}} .
$$

The four-momentum transfer is $Q^{\mu}=k^{\mu}-k^{\prime \mu}=(\omega, \boldsymbol{q})$, with $Q^{2}=\omega^{2}-q^{2}$. The factor $\mathcal{F}^{2}$ comes from the contraction between the lepton and the hadron tensor and contains all the relevant nuclear structure information. $\mathcal{F}^{2}$ can be decomposed into a charge-charge $(C C)$, charge-longitudinal $(C L)$, longitudinal-longitudinal $(L L)$ and two transverse $\left(T, T^{\prime}\right)$ responses, i.e.,

$$
\begin{aligned}
\mathcal{F}_{+(-)}^{2}= & V_{C C} R_{C C}+2 V_{C L} R_{C L}+V_{L L} R_{L L}+V_{T} R_{T} \\
& +2 \chi V_{T^{\prime}} R_{T^{\prime}},
\end{aligned}
$$

where $\chi=+1(-1)$ in the case of neutrino (antineutrino) scattering. The coefficients $V$ are given by

$$
\begin{aligned}
V_{C C} & =1-\delta^{2} \tan ^{2}(\bar{\vartheta} / 2), \quad V_{C L}=\frac{\omega}{q}+\frac{\delta^{2}}{\rho^{\prime}} \tan ^{2}(\bar{\vartheta} / 2), \\
V_{L L} & =\left(\frac{\omega}{q}\right)^{2}+\left(1+\frac{2 \omega}{q \rho^{\prime}}+\delta^{2} \rho\right) \delta^{2} \tan ^{2}(\bar{\vartheta} / 2) \\
V_{T} & =\tan ^{2}(\bar{\vartheta} / 2)+\frac{\rho}{2}-\frac{\delta^{2}}{\rho^{\prime}}\left(\frac{\omega}{q}+\frac{\rho \rho^{\prime} \delta^{2}}{2}\right) \tan ^{2}(\bar{\vartheta} / 2), \\
V_{T^{\prime}} & =\frac{1}{\rho^{\prime}}\left(1-\frac{\omega \rho^{\prime} \delta^{2}}{q}\right) \tan ^{2}(\bar{\vartheta} / 2),
\end{aligned}
$$

where

$$
\delta=\frac{m}{\sqrt{\left|Q^{2}\right|}}, \quad \rho=\frac{\left|Q^{2}\right|}{q^{2}}, \quad \rho^{\prime}=\frac{q}{E_{v}+\varepsilon^{\prime}} .
$$

The weak response functions are given by

$$
\begin{aligned}
R_{C C} & =W^{00}, R_{C L}=-\left(W^{03}+W^{30}\right) / 2, R_{L L}=W^{33}, \\
R_{T} & =W^{11}+W^{22}, R_{T^{\prime}}=-i\left(W^{12}-W^{21}\right) / 2,
\end{aligned}
$$

in terms of the components of the hadron tensor, which is given by suitable bilinear products of the transition matrix elements of the nuclear many-body charged-current operator $\hat{J}^{\mu}$ between the initial state $\left|\Psi_{0}\right\rangle$ of the nucleus, of energy $E_{0}$, and the final states $\left|\Psi_{f}\right\rangle$, of energy $E_{f}$, both eigenstates of the $(A+1)$-body Hamiltonian $H$, as

$$
\begin{aligned}
W^{\mu \nu}(q, \omega)= & \sum_{i} \int_{f}\left\langle\Psi_{f}\left|\hat{J}^{\mu}(\boldsymbol{q})\right| \Psi_{0}\right\rangle \\
& \times\left\langle\Psi_{0}\left|\hat{J}^{\nu \dagger}(\boldsymbol{q})\right| \Psi_{f}\right\rangle \delta\left(E_{0}+\omega-E_{f}\right),
\end{aligned}
$$

involving an average over the initial states and a sum over the undetected final states. The sum runs over the scattering states corresponding to all of the allowed asymptotic configurations and includes possible discrete states.

In the QE region the scattering is described in the oneboson exchange approximation and in the relativistic impulse 
approximation (RIA) by the sum of incoherent processes involving only one nucleon scattering, i.e., the scattering occurs with only one nucleon, which is subsequently emitted, while the remaining nucleons of the target behave as spectators. Within the RIA the nuclear current operator is assumed to be the sum of single-nucleon currents $j^{\mu}$, corresponding to the weak CC operator

$$
\begin{aligned}
j^{\mu}= & {\left[F_{1}^{V}\left(Q^{2}\right) \gamma^{\mu}+i \frac{\kappa}{2 m_{N}} F_{2}^{V}\left(Q^{2}\right) \sigma^{\mu \nu} Q_{\nu}\right.} \\
& \left.-G_{A}\left(Q^{2}\right) \gamma^{\mu} \gamma^{5}+F_{P}\left(Q^{2}\right) Q^{\mu} \gamma^{5}\right] \tau^{ \pm},
\end{aligned}
$$

where $\tau^{ \pm}$are isospin operators, $\kappa$ is the anomalous part of the magnetic moment, and $\sigma^{\mu \nu}=(i / 2)\left[\gamma^{\mu}, \gamma^{\nu}\right] . F_{1}^{V}$ and $F_{2}^{V}$ are the isovector Dirac and Pauli nucleon form factors, which are related to the corresponding electromagnetic form factor by the conservation of the vector current. $G_{A}$ and $F_{P}$ are the axial and induced pseudoscalar form factors which are usually parametrized as

$$
\begin{aligned}
G_{A} & =\frac{g_{A}}{\left(1+Q^{2} / M_{A}^{2}\right)^{2}}, \\
F_{P} & =\frac{2 m_{N} G_{A}}{m_{\pi}^{2}+Q^{2}},
\end{aligned}
$$

where $g_{A} \simeq 1.267, m_{\pi}$ is the pion mass, $m_{N}$ is the nucleon mass, and $M_{A} \simeq 1.03 \mathrm{GeV}$ is the axial mass [46-48].

Within the RIA framework and under the assumption of a shell-model description for nuclear structure, the components of the hadron tensor are obtained from the sum, over all the single-particle (s.p.) shell-model states, of the squared absolute value of the transition matrix elements of the single-nucleon current

$$
\left\langle\chi_{E}^{(-)}(E)\left|\hat{J}^{\mu}(\boldsymbol{q})\right| \varphi_{n}\right\rangle
$$

where $\chi_{E}^{(-)}(E)$ is the scattering state of the emitted nucleon and the overlap $\varphi_{n}$ between the ground state of the target $\left|\Psi_{0}\right\rangle$ and the final state $|n\rangle$ of the residual nucleus is a s.p. shell-model state. In this work the bound nucleon states $\varphi_{n}$ are taken as self-consistent Dirac-Hartree solutions derived within a RMF approach using a Lagrangian containing $\sigma, \omega$, and $\rho$ mesons [49-53].

Different prescriptions are used to calculate the relativistic s.p. scattering wave functions. In the relativistic plane-wave impulse approximation (RPWIA), FSI between the outgoing nucleon and the residual nucleus are neglected. In the relativistic distorted-wave impulse approximation (RDWIA) FSI effects are accounted for by solving the Dirac equation with strong relativistic scalar and vector optical potentials. This approach has been very successfull in describing exclusive $\left(e, e^{\prime} p\right)$ data [11-15,54,55], but it would be inconsistent for the inclusive scattering, where all the inelastic channels should be retained and the total flux, although redistributed among all possible channels due to FSI, must be conserved. In the RDWIA (with complex potentials) the flux is not conserved and the inclusive $\left(e, e^{\prime}\right)$ cross section is underestimated [23,56-58]. A simple way of estimating the inclusive response within the RIA is to use purely real potentials. In a first approach, the imaginary part of the phenomenological relativistic energydependent optical potentials [59] is set to zero, thus retaining in the calculations only the real part. In a second approach, the scattering states are described by using the same real scalar and vector RMF potentials considered in the description of the initial bound state $\varphi_{n}$. We refer to these two different FSI descriptions as real relativistic optical potential (rROP) and RMF calculations, respectively.

We note that the rROP conserves the flux and thus it is inconsistent with the exclusive process, where the final state includes only the elastic rescattering of the knocked-out nucleon and thus a complex optical potential adjusted to elastic proton scattering data must be used. Moreover, the use of a real optical potential is unsatisfactory from a theoretical point of view, since it is an energy-dependent potential, reflecting the different contribution of open inelastic channels for each energy. Dispersion relation then dictates that the potential should have a nonzero imaginary term [60]. On the other hand, the RMF model is based on the use of the same strong energy-independent real potential for both bound and scattering states, so that it fulfills the dispersion relation [60] and, further, it maintains the continuity equation.

In a different approach, Green's-function techniques may be exploited to derive the inclusive response. This formalism allows us to reconstruct the flux into nonelastic channels in the case of inclusive scattering, starting from the complex (scalar and vector) optical potential, which describes elastic-scattering data. The components of the hadron tensor are written in terms of the s.p. optical model Green's function. This is the result of suitable approximations, such as the assumption of a one-body current and subtler approximations related to the impulse approximation. The explicit calculation of the s.p. Green's function is avoided by using its spectral representation, which is based on a biorthogonal expansion in terms of a non-Hermitian optical potential $\mathcal{H}$ and of its Hermitian conjugate $\mathcal{H}^{\dagger}$. Calculations require matrix elements of the same type as the RDWIA ones in Eq. (12), but involve eigenfunctions of both $\mathcal{H}$ and $\mathcal{H}^{\dagger}$, where the different sign of the imaginary part gives absorption in one case and gain of flux in the other case. Thus in the sum over $n$ the total flux is redistributed and conserved. This relativistic Green's-function model allows for a consistent treatment of FSI in the exclusive and in the inclusive scattering and gives a good description of $\left(e, e^{\prime}\right)$ data [22,23]. Detailed discussions of the RGF model can be found in Refs. [22-26].

\section{A. Scaling at the quasielastic peak}

Scaling arguments applied to neutrino reactions were presented in Ref. [5], where a phenomenological "superscaling" approach, denoted as SuSA, was proposed based on the assumed universality of the scaling function for electromagnetic and weak interactions. Hence the phenomenological scaling function extracted from the analysis of electron-scattering data was used in order to make "model-independent" predictions for neutrino-nucleus cross sections. The universal scaling behavior is highly related to the single-boson exchange approximation and this justifies that both neutrino and electron 
scattering in $\mathrm{QE}$ conditions exhibit to a large extent similar scaling. A different way to analyze the problem consists of making use of a specific model that works properly in the description of electron-scattering data, and apply it to neutrino reactions. In this case, although cross sections are "model dependent," theoretical scaling functions corresponding to neutrinos can be derived and compared directly with the theoretical functions for electrons (obtained in the same model) and also with the experimental scaling function. Thus the universality property can be tested within the framework of a specific model.

In this work we apply scaling arguments to the RMF and RGF models. Results are shown in the next section. The usual procedure to get the scaling function has been considered, i.e., dividing the inclusive cross sections (1) by the appropriate single-nucleon charged-current $v N$ elastic cross section weighted by the corresponding proton/neutron number involved in the process. The scaling function $f\left(\psi^{\prime}\right)$ obtained with this procedure depends on the dimensionless scaling variable $\psi^{\prime}(q, \omega)$ extracted from the RFG analysis that incorporates the typical momentum scale for the selected nucleus $[4,20]$. The explicit expressions for the CC singlenucleon responses have already been presented in Refs. [5,6]. In these single-nucleon responses, effects coming from the motion of the nucleons in the nucleus have been considered only up to first order in the Fermi momentum $\eta_{F} \equiv k_{F} / m_{N}$. The use of the full results for the non-Pauli blocked regime, as shown in [6], leads to very minor differences without modifying the discussion and general conclusions presented in the work.

\section{RESULTS AND DISCUSSION}

In this section the numerical results of the different relativistic models developed by the Pavia and the MadridSevilla groups to describe FSI in the inclusive quasielastic $\mathrm{CC}$ neutrino-nucleus scattering are considered. As a first step results obtained by the two groups in the RPWIA and rROP approaches are compared to check the consistency of the numerical programs when calculations are carried out under the same conditions. Then the results corresponding to the RMF model developed by the Madrid-Sevilla group and the RGF model developed by the Pavia group are compared. The comparison is performed for the ${ }^{12} \mathrm{C}\left(v_{\mu}, \mu^{-}\right)$cross section and scaling function calculated in different kinematics which are representative of the kinematical range where QE scattering is expected to give the main contribution to the inclusive neutrino-nucleus scattering. Pion-production events may play a significant role and increase their contribution with larger values of the energy transfer. However, its contribution for the CCQE MiniBooNE data is expected to be very minor: no pions in the final state are detected. Antineutrino-induced scattering can also be calculated, but it is not considered in this paper. All the calculations are performed using the same relativistic initial-state wave functions, which are taken as Dirac-Hartree solutions of a relativistic Lagrangian written in the context of a relativistic mean-field theory with the NLSH parametrization [51-53].
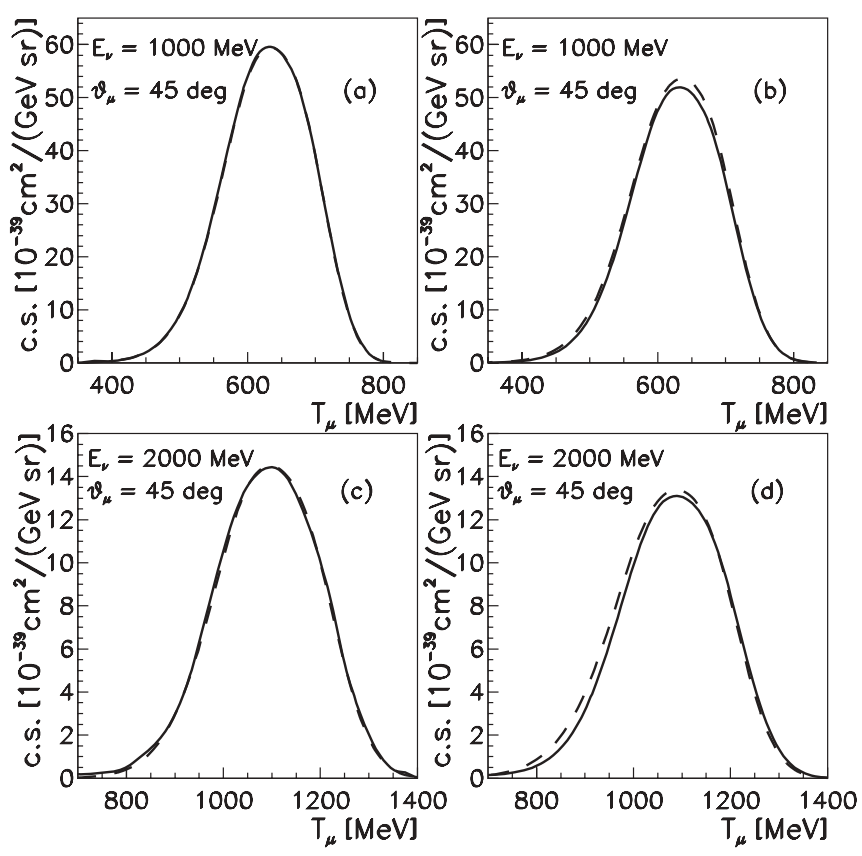

FIG. 1. Differential cross section of the ${ }^{12} \mathrm{C}\left(v_{\mu}, \mu^{-}\right)$reaction as a function of the kinetic energy of the outgoing muon $T_{\mu}$ for two values of the incident neutrino energy, i.e., $E_{\nu}=1000$ and $2000 \mathrm{MeV}$, and fixed muon scattering angle $\vartheta_{\mu}=45^{\circ}$, calculated by the Pavia (solid lines) and the Madrid-Sevilla (dashed lines) groups with RPWIA [(a) and (c)] and rROP [(b) and (d)].

\section{A. Differential cross section}

The ${ }^{12} \mathrm{C}\left(v_{\mu}, \mu^{-}\right)$cross section calculated by the Pavia and the Madrid-Sevilla groups in RPWIA and in rROP for two values of the incident neutrino energy, i.e., $E_{v}=1000$ and $2000 \mathrm{MeV}$, and a fixed muon scattering angle $\vartheta_{\mu}=45^{\circ}$ are compared in Fig. 1. Almost identical results are obtained in RPWIA. In rROP the two results are very similar, up to a few percent. A similar agreement between the RPWIA and rROP results of the Pavia and Madrid-Sevilla groups was already found in Ref. [22] for the inclusive QE electron scattering. The comparison in Fig. 1 is an important and necessary benchmark of our independent computer programs, which allows us to estimate the numerical uncertainties and gives enough confidence on the reliability of both calculations.

Cross sections evaluated with the RMF and RGF models for the kinematics with $E_{v}=1000,1500$, and $2000 \mathrm{MeV}$ and $\vartheta_{\mu}=45^{\circ}$ are presented in Fig. 2. In the case of the RGF approach two different parametrizations for the relativistic optical potential have been used: the energy-dependent and $A$-dependent EDAD1 and the energy-dependent but $A$ independent EDAI-12C complex phenomenological potentials of [59], which are fitted to proton elastic-scattering data on several nuclei in an energy range up to $1040 \mathrm{MeV}$. In the figures the results obtained with EDAD1 and EDAI-12C are denoted as GF1 and GF-EDAI, respectively. We note that all the RGF results presented here contain the contribution of both terms of the hadron tensor in Eq. (25) of Ref. [24], while only the contribution of the first term was included in the numerical results presented in Ref. [24]. The second term is entirely due 

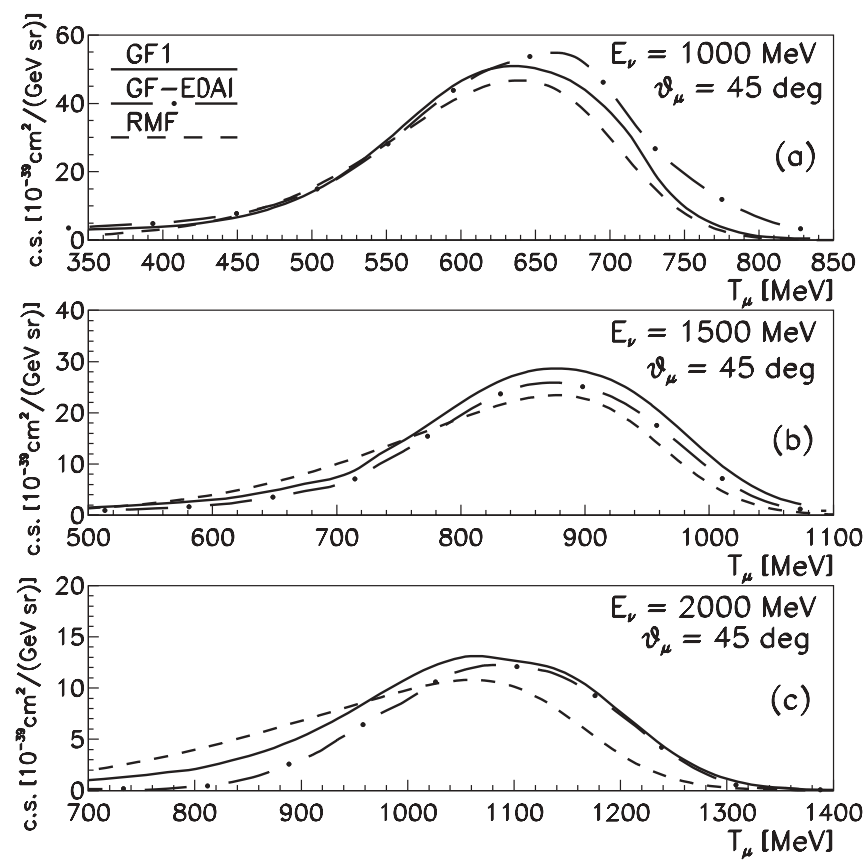

FIG. 2. Differential cross section of the ${ }^{12} \mathrm{C}\left(v_{\mu}, \mu^{-}\right)$reaction for three values of the incident neutrino energy, i.e., $E_{v}=1000$ (a), 1500 (b), and $2000 \mathrm{MeV}$ (c), and $\vartheta_{\mu}=45^{\circ}$. The solid and long dot-dashed lines are the RGF results calculated with the two different optical potentials EDAD1 and EDAI-12C. The dashed lines are the results of the RMF model.

to the imaginary part of the optical potential and vanishes if only the real part is considered. The calculation of this term is a complicated and time consuming task that requires integration over all the eigenfunctions of the continuum spectrum of the optical potential. Indeed the first term gives in general the main contribution. The contribution of the second term depends on kinematics and becomes more important for higher values of the energy transfer, i.e., higher energies for the optical potential. Although small and even negligible in many situations, the second term can be very important in particular kinematic conditions and in general cannot be neglected.

To make the comparison of the different models clearer, we compare in Fig. 3 the RMF and RGF results directly with the RPWIA and rROP ones. We note that in these kinematics, which are similar to those actually explored at present neutrino experiment facilities, the momentum transfer $q$ is not fixed and its value around the peak of the cross section is usually large, i.e., $q \approx 700 \mathrm{MeV} / c$ for $E_{v}=1000 \mathrm{MeV}$, $q \approx 1100 \mathrm{MeV} / c$ for $E_{v}=1500 \mathrm{MeV}$, and $q \approx 1400 \mathrm{MeV} / c$ for $E_{v}=2000 \mathrm{MeV}$. The shape of the RMF cross section shows an asymmetry, with a long tail extending toward lower values of the energy of the final muon, i.e., higher values of $\omega$, which is due to the strong energy-independent scalar and vector potentials present in the RMF approach. The asymmetry increases with larger incident neutrino energies. In the case of the RGF cross sections the asymmetry toward higher $\omega$ and $E_{v}$ is less significant but still visible. Almost no asymmetry is found for the RPWIA and rROP cross sections. We note that in the present calculations, which are
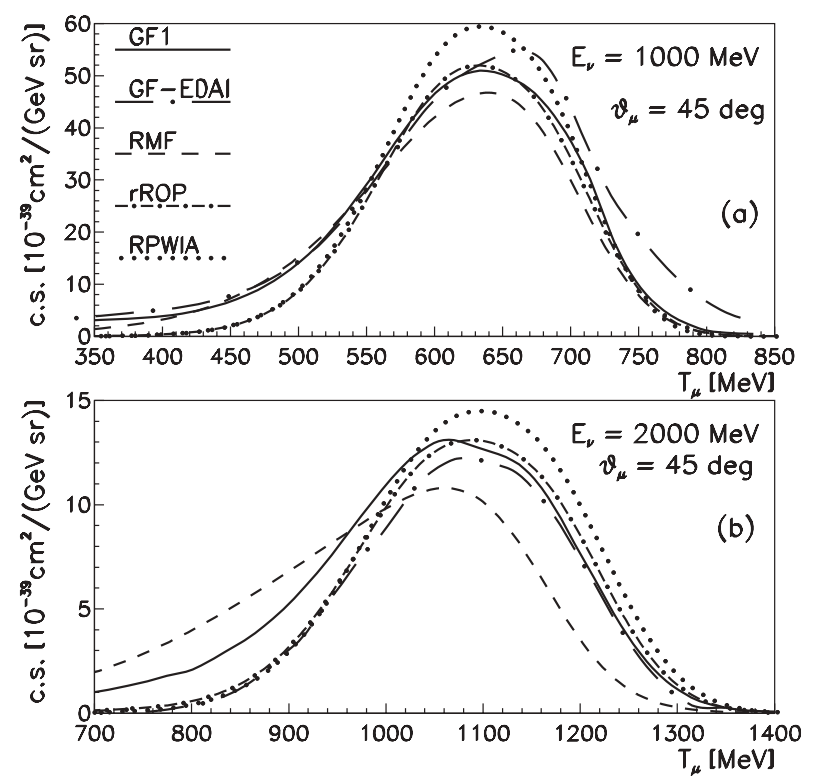

FIG. 3. Differential cross section of the ${ }^{12} \mathrm{C}\left(v_{\mu}, \mu^{-}\right)$reaction for $E_{v}=1000$ (a) and $2000 \mathrm{MeV}$ (b) and scattering angle $\vartheta_{\mu}=45^{\circ}$. The solid, long dot-dashed, and dashed lines are the same as in Fig. 2. The dot-dashed and dotted lines are the rROP and the RPWIA results, respectively.

performed in kinematics different from those considered in Ref. [24], significant differences are obtained between the rROP and RGF results. These differences stress the important role played by the imaginary part of the optical potential, which in the RGF model affects both terms of the hadron tensor. The GF1 and GF-EDAI cross sections have somewhat different shapes that are particularly visible for low $\omega$ at $E_{v}=1000 \mathrm{MeV}$ and for higher $\omega$ at $E_{v}=2000 \mathrm{MeV}$. These differences are essentially due to the imaginary part of the ROP that is sensitive to the particular parametrization considered. The real terms of the phenomenological ROP are very similar for different parametrizations and give very similar results.

The differences between the results of the GF1 and GFEDAI cross sections depend on the energy and momentum transfer, and are directly linked to the specific structure of the energy-dependent relativistic optical potentials adopted in the RGF model. The differences between the RMF and RGF results increase with the energy and momentum transfer. The larger differences seen for the largest value of $E_{v}$, not only between the RMF and RGF models, but also between the two RGF results, is simply an indication of the difference in the ingredients of these calculations.

To perform a more direct comparison between our neutrinoand electron-scattering calculations of Ref. [22], we present in Fig. 4 our cross sections calculated for a fixed value of the incident neutrino energy, $E_{v}=1000 \mathrm{MeV}$, and two values of the momentum transfer, i.e., $q=500$ and $1000 \mathrm{MeV} / c$. Also in these kinematics the shape of the RMF cross section shows an asymmetry with a tail extending toward higher values of $\omega$. An asymmetric shape toward higher $\omega$ is shown also by the RGF cross sections, while no visible asymmetry is given by the RPWIA and rROP results. Also in these kinematics significant 

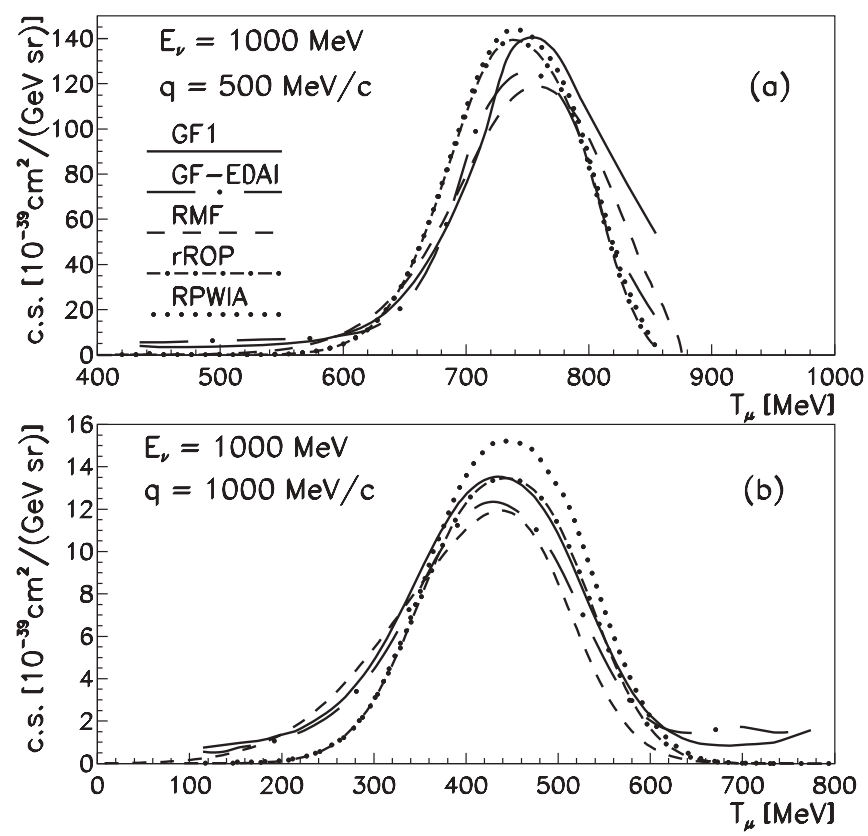

FIG. 4. Differential cross section of the ${ }^{12} \mathrm{C}\left(v_{\mu}, \mu^{-}\right)$reaction for $E_{v}=1000 \mathrm{MeV}$ and $q=500 \mathrm{MeV} / c$ (a) and $1000 \mathrm{MeV} / c$ (b). Line convention as in Fig. 3.

differences are obtained between the RGF and rROP cross sections.

The behavior of the RMF and RGF results as a function of $q$ and $\omega$ is connected to the different relativistic potentials used in the models. The RMF model uses the effective mean field, which reproduces the saturation behavior of nuclear matter and the properties of the ground state of nuclei. On the other hand, the RGF model uses the phenomenological optical potentials fitted to elastic proton-nucleus scattering. The lost of elastic flux into inelastic channels caused by the imaginary term of these potentials is recovered for inclusive scattering in the RGF making use of dispersion relations. As already shown for $\left(e, e^{\prime}\right)$ reactions [22], the RGF yields a larger cross section than the RMF. The latter can be considered as an estimation of the the pure nucleonic contribution. On the contrary, the RGF may account for in a phenomenological way additional effects due to non-nucleonic degrees of freedom, more important with increasing transferred momentum. Notice that both the RGF and the RMF yield predictions within a few percent for low- $q$ [Figs. 2(a) and 3(a)], while as $q$ goes up the RGF yields increasingly larger cross sections than RMF. This may reflect the influence of the pionic degrees of freedom.

The results obtained with GF1 and GF-EDAI are consistent with the general behavior of the phenomenological energydependent relativistic optical potentials and the differences are basically due to their different imaginary part, that includes the overall effect of the inelastic channels and is not univocally determined by the elastic phenomenology.

The results displayed in Fig. 4 present some differences with respect to the corresponding cross sections of the inclusive electron scattering shown in Ref. [22], which are calculated with the same models for FSI and at the same values of the momentum transfer. In both cases the differences between the results of the different models are generally larger for increasing value of the momentum transfer. For neutrino scattering, however, such a behavior is less evident and clear. In particular, the GF1 cross section at $q=1000 \mathrm{Mev} / c$ does not show the strong enhancement in the region of the maximum that was found for the $\left(e, e^{\prime}\right)$ calculations of Ref. [22], where the GF1 result was even larger than the RPWIA one. In the case of neutrino scattering the RGF results in the region of the maximum are generally larger than the RMF ones, but smaller than the RPWIA cross sections.

In spite of many similarities, inclusive electron scattering and CC neutrino-nucleus scattering are two different processes and caution should be drawn on their comparison. We note that the cross sections shown in Fig. 4 are calculated with same incident lepton energy and the same momentum transfer as in the $\left(e, e^{\prime}\right)$ calculations of Ref. [22]. Nevertheless, the muon mass gives in the case of $\mathrm{CC}$ neutrino scattering a different kinematics, with different values of the energy transfer and, as a consequence, of the energies of the outgoing nucleon. This means that in the RGF model the optical potential is calculated for electron and neutrino scattering at different energies. To clarify this point and reproduce the kinematics of electron scattering, we have performed some calculations for the $\left(v, \mu^{-}\right)$reaction with vanishing muon mass. The main difference with respect to the calculations shown in Fig. 4 is a shift of the cross section, by about $100 \mathrm{MeV}$, toward higher values of $T_{\mu}$, without any significant change in the shape or in the strength.

The most important difference between electron and neutrino scattering at the Feynman amplitude level is the exchanged vector boson: a virtual photon probing the electromagnetic current in electron scattering, and a $W^{ \pm}$probing the weak current in CC neutrino-nucleus scattering. Thus whereas for the $\left(e, e^{\prime}\right)$ process all nucleons (protons and neutrons) in the nucleus contribute, in the case of CC neutrino (antineutrino) scattering only the neutrons (protons) in the target contribute to the inclusive cross section. Moreover, whereas the $R_{L}$ and $R_{T}$ responses in CC neutrino induced scattering are given by the sum of pure vector-vector $(V V)$ and axial-axial $(A A)$ terms (see, e.g., Refs. [5,6]), the $R_{T^{\prime}}$ response comes from the interference between the vector $(V)$ and axial $(A)$ terms in the neutrino $\mathrm{CC}$ operator. In the electron scattering case, where both isoscalar and isovector form factors enter the responses, the cross section is the sum of the longitudinal and transverse responses.

The different currents and their possible interplay with the other ingredients of the models do not allow an easy comparison between the results of electron and neutrino scattering. The numerical differences between the RGF results for electron and neutrino scattering can mainly be ascribed to the combined effects of the weak current, in particular, its axial term, and the imaginary part of the relativistic optical potential. We have checked that these effects can explain the fact that in neutrino scattering the GF1 result does not give the strong enhancement in the region of the maximum of the cross section that was found for the $\left(e, e^{\prime}\right)$ calculations of Ref. [22]. 

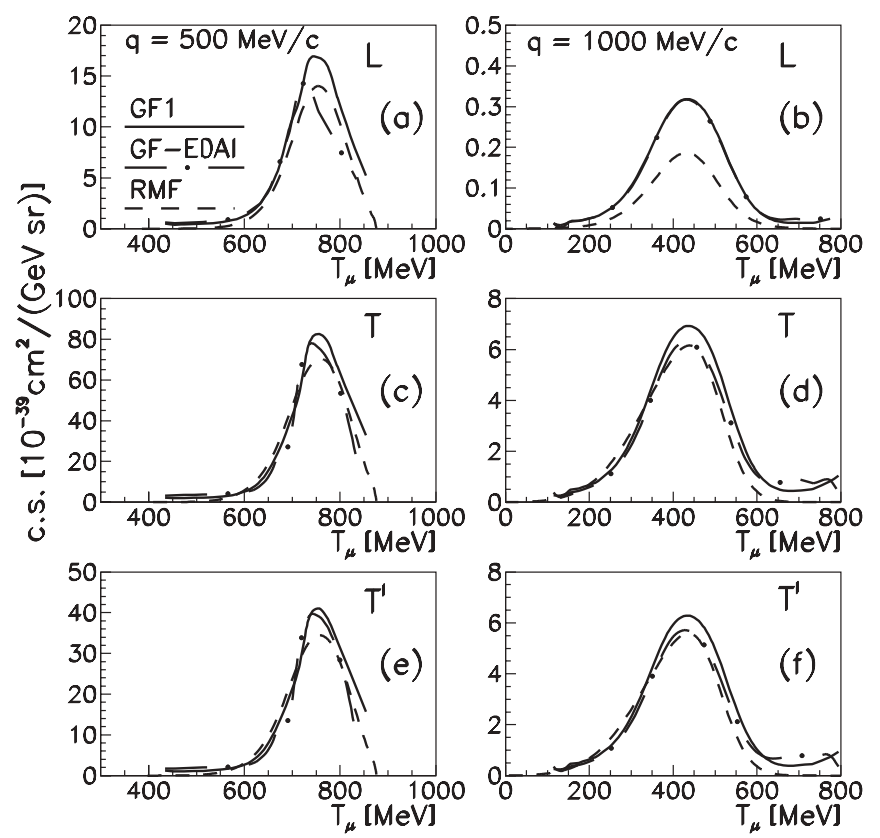

FIG. 5. $L, T$, and $T^{\prime}$ components of the cross section of the ${ }^{12} \mathrm{C}\left(v_{\mu}, \mu^{-}\right)$reaction for $E_{v}=1000 \mathrm{MeV}$ and $q=500 \mathrm{MeV} / c$ [panels (a), (c), and (d) in the left column] and $1000 \mathrm{MeV} / c$ [panels (b), (d), and (e) in the right column]. Line convention as in Fig. 2.

As a further example, we compare in Fig. 5 the RMF and RGF results for the longitudinal, transverse, and interference axial-vector contributions to the cross section. The sum of these three terms gives the corresponding cross sections in Fig. 4. Here, by longitudinal contribution to the cross section we mean the sum of the $R_{L L}, R_{C L}$, and $R_{C C}$ responses, defined in Eq. (7), each one multiplied by the appropriate kinematical factors of Eq. (5). In general, the RMF and RGF results as a function of $q$ and $\omega$ show the same behavior as the cross sections in Fig. 4 and, again, are related to the different relativistic potentials used in the models. The $T$ and $T^{\prime}$ components are similar for all cases: at $q=500 \mathrm{MeV} / c$ both RGF results are larger than the RMF ones in the peak region, while at $q=1000 \mathrm{MeV} / c$ the RMF and GF-EDAI results are very similar and lower than GF1. The longitudinal cross section has a different behavior: at $q=500 \mathrm{MeV} / c$ the GF-EDAI result is close to RMF and lower than GF1, while at $q=1000 \mathrm{MeV} / c$ it overlaps GF1 and they both are larger than RMF. At $q=500 \mathrm{MeV} / c$ all the RGF and RMF calculations give the $T$ cross section approximately two times larger than the $T^{\prime}$ one. In addition, the longitudinal contribution, although smaller, cannot be neglected; in this case the relative difference between the GF1 and GF-EDAI longitudinal components is large and has visible effects in the cross sections of Fig. 4. At $q=1000 \mathrm{MeV} / c$ the $T$ and $T^{\prime}$ components have similar strengths, whereas the longitudinal response gives only a very small contribution to the cross section. In the electron scattering case, at $q=500 \mathrm{MeV} / c$ the longitudinal and transverse components have similar strength whereas at $q=1000 \mathrm{MeV} / c$ the transverse response is much more important (see, e.g., Refs. [22,23]).

\section{B. Scaling functions}

The effects already discussed for the differential cross sections are also present in the scaling functions. Here we compare results for the scaling function extracted from the differential cross section $f\left(\psi^{\prime}\right)$ using the same descriptions for the final-state interactions already considered for the cross sections. The transverse responses $R_{T}$ and $R_{T^{\prime}}$ are the leading terms for the total cross section whereas the longitudinal contribution $R_{L}$ is usually small; the corresponding scaling function $f_{T}\left(\psi^{\prime}\right)$ and $f_{T^{\prime}}\left(\psi^{\prime}\right)$ are very similar, up to a few percent, and, moreover, they are similar to the scaling function $f\left(\psi^{\prime}\right)$ from the cross section, i.e., scaling of zero kind is verified. Thus we do not show $f_{T}\left(\psi^{\prime}\right)$ and $f_{T^{\prime}}\left(\psi^{\prime}\right)$ and present our results only for $f\left(\psi^{\prime}\right)$. The longitudinal contribution leads to a scaling function $f_{L}\left(\psi^{\prime}\right)$, which may depart significantly from $f\left(\psi^{\prime}\right)$. However, one should be cautious because the longitudinal contribution to inclusive neutrino-nucleus cross sections can be almost negligible in some kinematics compared with the transverse $T$ and $T^{\prime}$ ones.

As a first step, we have compared the scaling function $f\left(\psi^{\prime}\right)$ obtained by the Pavia and Madrid-Sevilla groups in RPWIA and rROP for three values of the neutrino energy, $E_{v}=1000,1500$, and $2000 \mathrm{MeV}$, and $\vartheta_{\mu}=45^{\circ}$. Our results are almost coincident and, in addition to the cross-section results in Fig. 1, confirm the consistency of the numerical codes when calculations are performed under the same conditions. We do not show these results for simplicity.

In Figs. 6 and 7 we compare the scaling function $f\left(\psi^{\prime}\right)$ evaluated with different models and for the same kinematical conditions as in Figs. 2 and 4, respectively, with the averaged QE phenomenological longitudinal scaling function extracted from the analysis of $\left(e, e^{\prime}\right)$ data [2-4]. As already shown in previous works [19-21], the RMF model produces an
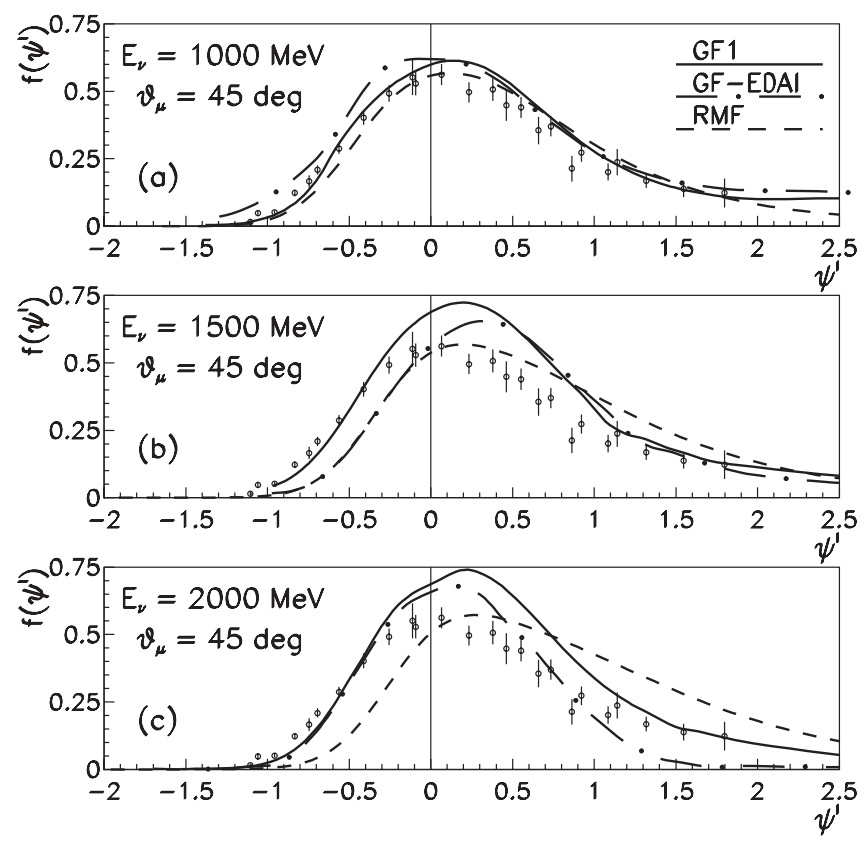

FIG. 6. Scaling function of the ${ }^{12} \mathrm{C}\left(v_{\mu}, \mu^{-}\right)$reaction in the same kinematics as in Fig. 2 compared with the averaged experimental scaling function. Line convention as in Fig. 2. 

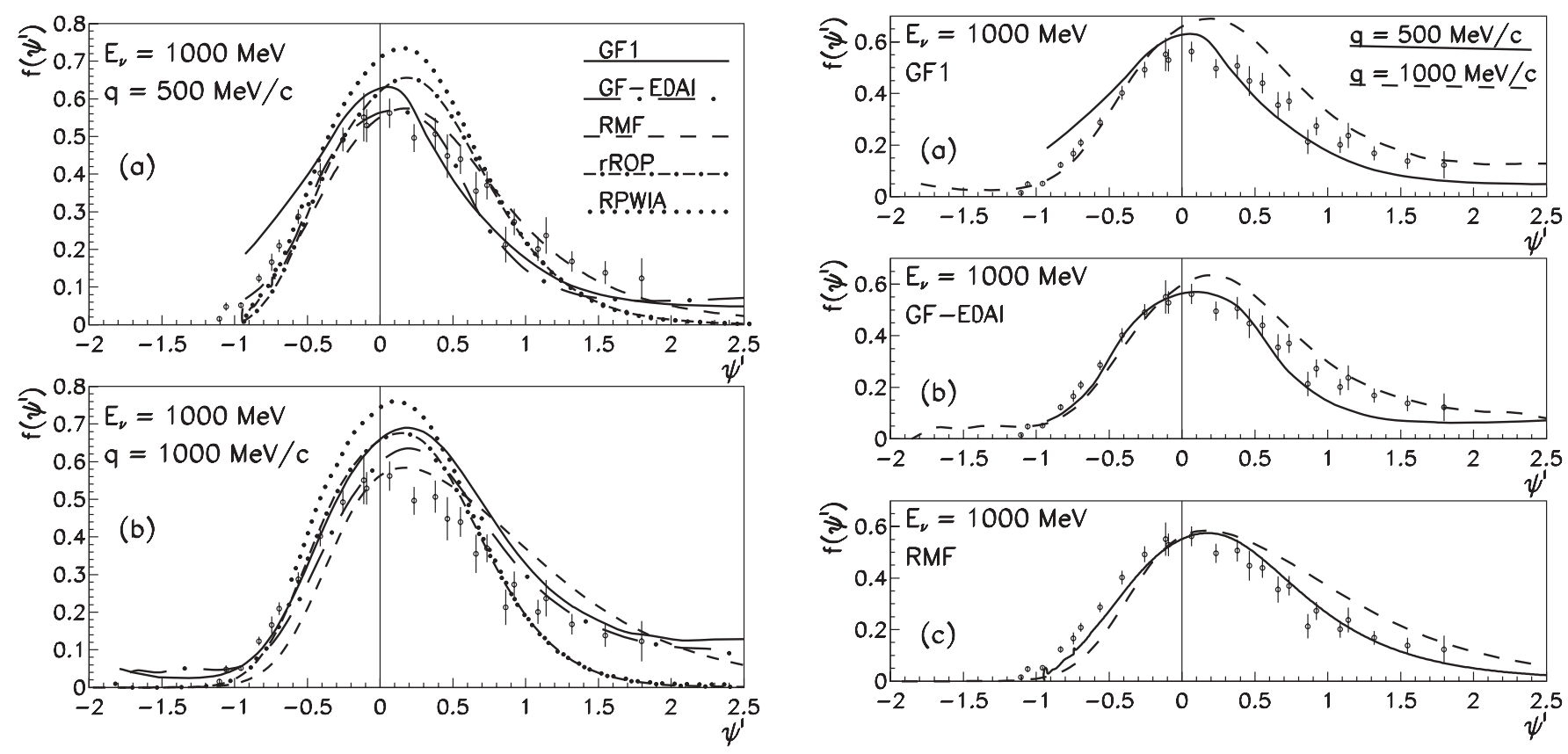

FIG. 7. Scaling function of the ${ }^{12} \mathrm{C}\left(v_{\mu}, \mu^{-}\right)$reaction for incident neutrino energy $E_{v}=1000 \mathrm{MeV}$ and $q=500 \mathrm{MeV} / c$ (a) and $1000 \mathrm{MeV} / c$ (b). Line convention as in Fig. 4.

asymmetric shape, with a long tail in the region with $\psi^{\prime}>0$, that follows closely the behavior of the phenomenological function. It has been noticed that the RMF approach is capable of explaining the asymmetric behavior of the phenomenolgical scaling function within the framework of the relativistic impulse approximation taking advantage of its strong relativistic scalar and vector potentials. The results with the RGF model in Fig. 6 are similar to those obtained with RMF at $E_{v}=$ $1000 \mathrm{MeV}$, while visible discrepancies appear at $E_{v}=1500$ and $2000 \mathrm{MeV}$, and in Fig. 7. As a general remark, these results for the scaling functions follow similar trends to those already applied to the behavior of the cross sections in Figs. 2 and 4, i.e., the differences between the RMF and RGF, and between the GF1 and GF-EDAI calculations, increase with larger energy and momentum transfer.

The asymmetric shape with a tail in the region of positive $\psi^{\prime}$ is obtained in both RMF and RGF models, which involve descriptions of FSI either with a strong energy-independent real potential or with a complex energy-dependent optical potential, respectively. The scaling functions corresponding to RPWIA and rROP, which are also presented in Fig. 7 for two values of the momentum transfer, do not show any significant asymmetric tail for $\psi^{\prime}>0$.

It has been already discussed [19-21] that the RMF model produces a scaling function $f\left(\psi^{\prime}\right)$ that is in accordance with the longitudinal scaling function extracted from the $\left(e, e^{\prime}\right)$ reaction and hence with the electron-scattering longitudinal data. This outcome reinforces the validity of the general assumption [20] to predict CC neutrino-nucleus cross sections from the phenomenological scaling function, and it is a noticeable fact that this is contrary to what one would expect, since neutrino reactions are totally dominated by the transverse $R_{T}$ and $R_{T^{\prime}}$ responses.

FIG. 8. Analysis of first kind scaling, $f\left(\psi^{\prime}\right)$ for $E_{v}=1000 \mathrm{MeV}$ and $q=500 \mathrm{MeV} / c$ (solid lines) and $1000 \mathrm{MeV} / c$ (dotted lines) with the GF1 (a), GF-EDAI (b), and RMF (c) models using the same results already displayed in Fig. 7.

In the case of the RGF model we apparently do not obtain a similar good agreement with the longitudinal scaling function $f_{L}\left(\psi^{\prime}\right)$ extracted from the $\left(e, e^{\prime}\right)$ results of Ref. [22]. This is clearly related to the fact that, at higher $q$ values, there is not such a strong enhancement of the maximum strength, particularly for GF1 as in Ref. [22]. However, taking advantage of this, the RGF model produces a scaling function in reasonable agreement with the averaged experimental scaling function.

An analysis of the scaling of first kind, i.e., independence of the momentum transfer, is illustrated in Fig. 8. The results are the same already shown in Fig. 7, but are presented in a different way. Each panel corresponds to a specific description of FSI (GF1, GF-EDAI, and RMF) and includes the results obtained at $q=500$ and $1000 \mathrm{MeV} / c$. The experimental $\left(e, e^{\prime}\right)$ data are compatible with a mild violation of the first-kind scaling, particularly in the positive $\psi^{\prime}$ region. In Refs. $[19,20]$ the scaling functions evaluated with the RPWIA and rROP models were shown to depend very mildly on the transferred momentum in the whole, positive and negative, $\psi^{\prime}$ region. In the case of the RMF approach, there is a slight shift in the region $\psi^{\prime}<0$ (slighter than for the longitudinal scaling function of electron scattering), whereas the model breaks scaling approximately at $30 \%$ level when $\psi^{\prime}>0$. Similar results are obtained with the RGF models, where a small shift in the region of negative $\psi^{\prime}$ also occurs, and scaling is broken for $\psi^{\prime}>0$. This scaling violation for $\psi^{\prime}>0$ is slightly larger with GF1 than GF-EDAI. However, the quality of the comparison between the experimental QE scaling function and the two RGF results is similar. Finally, the stronger scaling breakdown shown by RGF, compared to RMF, may reflect the effective presence of non-nuclenic contributions in the case of RGF. 


\section{SUMMARY AND CONCLUSIONS}

This work emerges from the collaboration between the Pavia and Madrid-Sevilla groups, and it extends the study presented in Ref. [22] where we focused on inclusive electron scattering reactions. Here we consider the case of chargedcurrent quasielastic neutrino-nucleus scattering processes. These studies are of great interest in connection with the recent data measured at MiniBooNE [27], and their "possible" impact on neutrino oscillations.

The kinematics of the MiniBooNE experiment involves neutrino energies up to $\sim 3 \mathrm{GeV}$. This implies that relativity is an essential ingredient; not only relativistic kinematics should be considered, but also nuclear dynamics and current operators should be described within a relativistic framework. The analysis performed by our groups involves a relativistic description of the reaction mechanism with strong scalar and vector potentials, thus providing a proper treatment of the neutrino scattering process of interest. However, before entering into a detailed analysis of the MiniBooNE experiment and its comparison with our theoretical results, which will be considered in a subsequent work, in this paper we restrict ourselves to a systematic comparison between the two approaches for different kinematics. As already mentioned in Ref. [22], the description of the final-state interactions constitutes a basic aspect in the analysis of the theoretical results and their comparison with data.

Following Ref. [22], we check the consistency of the numerical calculations by comparing results in the plane-wave limit (RPWIA) and also making use of the real part of the relativistic optical potentials (rROP). Almost identical results are obtained in RPWIA and very close in the case of rROP. This outcome is consistent with our previous analysis in electron scattering, and it reinforces the reliability of the calculations. Next we apply the two models considered in Ref. [22] to account for FSI, that is, the RMF, based on the use of the same relativistic mean-field potential for the bound and ejected nucleon wave functions, and the RGF approach, which treats FSI consistently in the inclusive and exclusive reactions. In the latter we have considered two different optical potentials, EDAD1 and EDAI-12C, which have been fitted to proton elastic-scattering data.

Contrary to RPWIA and rROP approaches, the differential cross sections obtained within RMF and RGF show a significant asymmetry with a tail extended to large values of the energy transfer (small muon kinetic energies). The amount of this asymmetry in the RMF approach increases with larger neutrino energies. On the contrary, its contribution is less significant in the RGF model, showing also important differences attached to the two optical potentials considered. These discrepancies are linked to the imaginary energy-dependent term in the ROP, which is very sensitive to the particular parametrization considered. It seems that optical potentials fitted only to elastic-scattering data are not well constrained when applied, within the RGF approach, to describe inclusive reactions. In addition to these effects introduced by the particular ROP considered, also significant differences emerge from the comparison between RMF and RGF, being larger for increasing transferred momentum. Although this general behavior was already present in the case of $\left(e, e^{\prime}\right)$ processes [22], the results for neutrino reactions show some distinct features to be discussed. In particular, the GF1 $\left(v, \mu^{-}\right)$cross section does not present the strong enhancement in the region of the maximum that was previously found for $\left(e, e^{\prime}\right)$ at $q=1000 \mathrm{MeV} / c$. This result has implications for the scaling function. As shown in Ref. [21], the function $f\left(\psi^{\prime}\right)$ obtained for CCQE neutrino reactions within the RMF model is in accordance with $f_{L}\left(\psi^{\prime}\right)$ from $\left(e, e^{\prime}\right)$. This is connected to the isospin content in the nucleon form factors. On the contrary, such an agreement does not occur for RGF. This would indicate that the universality property of the superscaling function is not entirely fulfilled within the RGF model.

Summarizing, we present a systematic analysis of cross sections and scaling properties for CCQE neutrino-nucleus scattering processes. We consider two different relativistic descriptions of the final-state interactions. Although both approaches respect scaling and superscaling behavior, some differences emerge, particularly concerning the comparison between the results obtained for electrons and neutrinos. The present results should be cautiously viewed due to the very different ingredients considered by RMF and RGF models; however, these may help in disentangling different physics aspects involved in the processes, with a special mention to the analysis of the MiniBooNE data and FSI effects. Work along this line is presently in progress.

\section{ACKNOWLEDGMENTS}

We are grateful to M. B. Barbaro and F. D. Pacati for useful discussions and for their valuable advice. This work was partially supported by DGI (Spain) under Contracts No. FIS2008-04189 and No. FPA2010-17142, the Spanish Consolider-Ingenio 2010 program CPAN (CSD2007-00042), and by the INFN-CICYT collaboration agreements FPA200803770-E and ACI2009-1053.
[1] D. B. Day, J. S. McCarthy, T. W. Donnelly, and I. Sick, Annu. Rev. Nucl. Part. Sci. 40, 357 (1990).

[2] T. W. Donnelly and I. Sick, Phys. Rev. Lett. 82, 3212 (1999).

[3] T. W. Donnelly and I. Sick, Phys. Rev. C 60, 065502 (1999).

[4] C. Maieron, T. W. Donnelly, and I. Sick, Phys. Rev. C 65, 025502 (2002).

[5] J. E. Amaro, M. B. Barbaro, J. A. Caballero, T. W. Donnelly, A. Molinari, and I. Sick, Phys. Rev. C 71, 015501 (2005).
[6] J. E. Amaro, M. B. Barbaro, J. A. Caballero, T. W. Donnelly, and C. Maieron, Phys. Rev. C 71, 065501 (2005).

[7] A. N. Antonov, M. V. Ivanov, M. K. Gaidarov, E. Moya de Guerra, J. A. Caballero, M. B. Barbaro, J. M. Udías, and P. Sarriguren, Phys. Rev. C 74, 054603 (2006).

[8] A. N. Antonov, M. V. Ivanov, M. B. Barbaro, J. A. Caballero, E. Moya de Guerra, and M. K. Gaidarov, Phys. Rev. C 75, 064617 (2007). 
[9] J. E. Amaro, M. B. Barbaro, J. A. Caballero, T. W. Donnelly, and J. M. Udías, Phys. Rev. C 75, 034613 (2007).

[10] C. R. Chinn, A. Picklesimer, and J. W. Van Orden, Phys. Rev. C 40, 790 (1989).

[11] J. M. Udías, P. Sarriguren, E. Moya de Guerra, E. Garrido, and J. A. Caballero, Phys. Rev. C 48, 2731 (1993); 51, 3246 (1995).

[12] J. M. Udías, P. Sarriguren, E. Moya de Guerra, and J. A. Caballero, Phys. Rev. C 53, R1488 (1996)

[13] J. M. Udías, J. A. Caballero, E. Moya de Guerra, J. R. Vignote, and A. Escuderos, Phys. Rev. C 64, 024614 (2001).

[14] A. Meucci, C. Giusti, and F. D. Pacati, Phys. Rev. C 64, 014604 (2001).

[15] A. Meucci, C. Giusti, and F. D. Pacati, Phys. Rev. C 64, 064615 (2001).

[16] A. Meucci, Phys. Rev. C 65, 044601 (2002).

[17] J. M. Udías, J. A. Caballero, E. Moya de Guerra, J. E. Amaro, and T. W. Donnelly, Phys. Rev. Lett. 83, 5451 (1999).

[18] M. C. Martínez, J. R. Vignote, J. A. Caballero, T. W. Donnelly, E. Moya de Guerra, and J. M. Udías, Phys. Rev. C 69, 034604 (2004).

[19] J. A. Caballero, J. E. Amaro, M. B. Barbaro, T. W. Donnelly, C. Maieron, and J. M. Udías, Phys. Rev. Lett. 95, 252502 (2005).

[20] J. A. Caballero, Phys. Rev. C 74, 015502 (2006).

[21] J. A. Caballero, J. E. Amaro, M. B. Barbaro, T. W. Donnelly, and J. M. Udías, Phys. Lett. B 653, 366 (2007).

[22] A. Meucci, J. A. Caballero, C. Giusti, F. D. Pacati, and J. M. Udías, Phys. Rev. C 80, 024605 (2009).

[23] A. Meucci, F. Capuzzi, C. Giusti, and F. D. Pacati, Phys. Rev. C 67, 054601 (2003).

[24] A. Meucci, C. Giusti, and F. D. Pacati, Nucl. Phys. A 739, 277 (2004).

[25] F. Capuzzi, C. Giusti, and F. D. Pacati, Nucl. Phys. A 524, 681 (1991).

[26] F. Capuzzi, C. Giusti, F. D. Pacati, and D. N. Kadrev, Ann. Phys. 317, 492 (2005).

[27] A. A. Aguilar-Arevalo et al. (MiniBooNE Collaboration), Phys. Rev. D 81, 092005 (2010).

[28] M. C. Martínez, P. Lava, N. Jachowicz, J. Ryckebusch, K. Vantournhout, and J. M. Udías, Phys. Rev. C 73, 024607 (2006).

[29] P. Lava, M. C. Martínez, J. Ryckebusch, J. A. Caballero, and J. M. Udías, Phys. Lett. B 595, 177 (2004).

[30] A. Meucci, C. Giusti, and F. D. Pacati, Nucl. Phys. A 773, 250 (2006).

[31] A. Meucci, C. Giusti, and F. D. Pacati, Phys. Rev. C 77, 034606 (2008).

[32] H. Kim, J. Piekarewicz, and C. J. Horowitz, Phys. Rev. C 51, 2739 (1995)

[33] C. Volpe, N. Auerbach, G. Colo, T. Suzuki, and N. Van Giai, Phys. Rev. C 62, 015501 (2000).

[34] J. Nieves, M. Valverde, and M. J. Vicente Vacas, Phys. Rev. C 73, 025504 (2006).
[35] A. V. Butkevich and S. A. Kulagin, Phys. Rev. C 76, 045502 (2007).

[36] A. V. Butkevich, Phys. Rev. C 82, 055501 (2010).

[37] O. Benhar, N. Farina, H. Nakamura, M. Sakuda, and R. Seki, Phys. Rev. D 72, 053005 (2005).

[38] O. Benhar and D. Meloni, Nucl. Phys. A 789, 379 (2007).

[39] C. Bleve, G. Co', I. De Mitri, P. Bernardini, G. Mancarella, D. Martello, and A. Surdo, Astropart. Phys. 16, 145 (2001); G. Co', C. Bleve, I. De Mitri, and D. Martello, Nucl. Phys. Proc. Suppl. 112, 210 (2002).

[40] M. Martini, M. Ericson, G. Chanfray, and J. Marteau, Phys. Rev. C 80, 065501 (2009).

[41] T. Leitner, L. Alvarez-Ruso, and U. Mosel, Phys. Rev. C 73, 065502 (2006).

[42] O. Buss, T. Leitner, U. Mosel, and L. Alvarez-Ruso, Phys. Rev. C 76, 035502 (2007).

[43] T. Leitner, O. Buss, L. Alvarez-Ruso, and U. Mosel, Phys. Rev. C 79, 034601 (2009).

[44] Y. Umino and J. M. Udias, Phys. Rev. C 52, 3399 (1995).

[45] J. D. Walecka, in Muon Physics, edited by V. H. Hughes and C. S. Wu (Academic, New York, 1975), Vol. II, p. 113.

[46] S. Galster et al., Nucl. Phys. B 32, 221 (1971).

[47] W. M. Alberico, S. M. Bilenky, and C. Maieron, Phys. Rep. 358, 227 (2002).

[48] V. Bernard, L. Elouadrhiri, and U. G. Meissner, J. Phys. G 28, R1 (2002).

[49] C. J. Horowitz and B. D. Serot, Nucl. Phys. A 368, 503 (1981); Phys. Lett. B 86, 146 (1979).

[50] B. D. Serot and J. D. Walecka, Adv. Nucl. Phys. 16, 1 (1986).

[51] W. Pöschl, D. Vretenar, and P. Ring, Comput. Phys. Commun. 103, 217 (1997).

[52] G. A. Lalazissis, J. König, and P. Ring, Phys. Rev. C 55, 540 (1997).

[53] M. M. Sharma, M. A. Nagarajan, and P. Ring, Phys. Lett. B 312, 377 (1993).

[54] J. J. Kelly, Adv. Nucl. Phys. 23, 75 (1996).

[55] S. Boffi, C. Giusti, F. D. Pacati, and M. Radici, Electromagnetic Response of Atomic Nuclei, Oxford Studies in Nuclear Physics Vol. 20 (Clarendon, Oxford, 1996); Phys. Rep. 226, 1 (1993).

[56] C. Maieron, M. C. Martínez, J. A. Caballero, and J. M. Udías, Phys. Rev. C 68, 048501 (2003).

[57] Y. Jin, D. S. Onley, and L. E. Wright, Phys. Rev. C 45, 1333 (1992).

[58] K. S. Kim and L. E. Wright, Phys. Rev. C 68, 027601 (2003); 72, 064607 (2005).

[59] B. C. Clark, in Proceedings of the Workshop on Relativistic Dynamics and Quark-Nuclear Physics, edited by M. B. Johnson and A. Picklesimer (John Wiley \& Sons, New York, 1986), p. 302; E. D. Cooper, S. Hama, B. C. Clark, and R. L. Mercer, Phys. Rev. C 47, 297 (1993).

[60] Y. Horikawa, F. Lenz, and N. C. Mukhopadhyay, Phys. Rev. C 22, 1680 (1980). 